\title{
Role of Regional Anaesthesia in Laparoscopy During COVID-19
}

\author{
Kaushik Bhattacharya ${ }^{1}$ (1)
}

Received: 14 May 2020 / Accepted: 29 May 2020/Published online: 6 June 2020

(C) Association of Surgeons of India 2020

Dear Sir,

"Regional anesthesia is like a lazy cheetah: it can be spotty and may not work; general anesthesia always works."

Regional anaesthesia (RA) was not regarded as a suitable anaesthesia for laparoscopic surgery because of the risk of aspiration, increased load of carbon dioxide, and shoulder tip pain. The main problem of RA is accelerated hypotension due to sympathetic blockade, ventilatory changes due to higher sensory levels required, and diaphragmatic irritation leading to shoulder tip pain along with increased surgical time due to the limitation of intra-abdominal pressure [1]. Nevertheless, it preserves respiratory function and avoids aerosolization and hence viral transmission; RA should be considered whenever surgery is planned for a suspected or confirmed COVID-19 patient undergoing laparoscopic procedures.

\section{Problems with General Anaesthesia}

In a recent report related to 138 confirmed COVID-19 cases, $41.3 \%$ were considered hospital acquired infection, and more than $70 \%$ were healthcare providers [2]. In anaesthesia practice, it is important to reduce exposure to patient's respiratory secretion and minimize the aerosol-generating procedures performed in general anaesthesia (GA) like bag mask ventilation, open airway ventilation, open airway suctioning, and endotracheal intubation. A systemic literature review and metaanalysis that evaluated the transmission of Severe Acute Respiratory Syndrome Corona Virus 1 (SARS-COV-1) to health care personnel in association with exposure to aerosol generating procedure found a significantly increased odds ratio of 6.6 [3]. The authors concluded that the ensemble of

Kaushik Bhattacharya

kbhattacharya10@yahoo.com

1 CAPFs Composite Hospital BSF Kadamtala, G616, Uttorayon, Matigara, Siliguri, West Bengal 734010, India personal protective equipment (PPE) used in the simulation may not fully prevent exposure of personnel performing endotracheal intubation in emergency department settings. GA in a COVID-19 patient is a high-risk procedure owing to the proximity of the health care workers to the patient's oropharynx, which carries a high viral load. Moreover, another situation, which poses a huge threat to ICU and operation theatre staff, is most often performed by anaesthesiologists, is cardiopulmonary resuscitation (CPR).

\section{Advantages of Regional Anaesthesia}

In order to avoid any airway manipulation during COVID-19, the use of RA may be preferred. There is general benefit of reduced pain and opioid consumption, reduced postoperative pulmonary complication, reduced postoperative nausea and vomiting, and reduced postoperative cognitive dysfunction and delirium during RA for laparoscopic surgery. The avoidance of airway instrumentation also prevents patient coughing during intubation and extubation [4]. RA has fewer effects on respiratory function and dynamics compared with GA with or without muscle paralysis. This relative preservation of respiratory function could theoretically reduce postoperative pulmonary complications in COVID-19 patients who may already have reduced respiratory function from COVID-19associated pneumonia or acute respiratory distress syndrome. However, this approach requires a co-operative patient, an experienced surgeon, and an enthusiastic anaesthesiologist ever prepared to supplement it with intravenous adjuncts and if needed with general anaesthesia. From these, we conclude that with proper application and with suitable improvements, regional anaesthesia has got the potential to emerge as the novel gold standard anaesthetic technique for elective laparoscopic surgeries [5].

Having a well-thought-out RA plan to manage infected patients in this new normal will ensure the best possible outcome for both the patient and the perioperative management team during laparoscopy. 


\section{Compliance with Ethical Standards}

Conflict of Interest The author declares that there is no conflict of interest.

\section{References}

1. Bajwa SJ, Kulshrestha A (2016) Anaesthesia for laparoscopic surgery: general vs regional anaesthesia. J Minim Access Surg 12:4-9

2. Luo M, Cao S, Wei L, Tang R, Hong S, Liu R, Wang Y (2020) Precautions for intubating patients with COVID-19. Anesthesiology 132:1616-1618
3. Weissman DN, de Perio MA, Radonovich LJ. COVID-19 and risks posed to personnel during endotracheal intubation. JAMA Published online April 27, 2020. doi:https://doi.org/10.1001/jama.2020.6627, 2027

4. Lie SA, Wong SW, Wong LT, Wong TGL, Chong SY (2020) Practical considerations for performing regional anesthesia: lessons learned from the COVID-19 pandemic. Can J Anaesth 24:1-8

5. Singh RK, Saini AM, Goel N, Bisht D, Seth A (2015) Major laparoscopic surgery under regional anesthesia: a prospective feasibility study. Med J Armed Forces India 71:126-131

Publisher's Note Springer Nature remains neutral with regard to jurisdictional claims in published maps and institutional affiliations. 